\title{
Le rôle d'un Maire de la ville dans la sécurisation de son entité administrative
}

\author{
Joseph KAZADI MPIANA*
}

\section{Résumé.}

La Ville de Lubumbashi est confrontée à une recrudescence sans précédent de l'insécurité. En sa qualité de première autorité administrative de la Ville, le Maire est tenu de procurer la sécurité à ses administrés en recourant notamment aux différentes mesures de police. L'article examine, entre autres, les défis se posant au Maire dans ses prérogatives de maintenir l'ordre public en général et la sécurité publique en particulier.

Sommaire : A. Introduction. B. Régionalisme politique et décentralisation en RDC. C. Le statut de la ville et du Maire dans l'ordonnancement juridique congolais. D. Le Maire face à l'impératif sécuritaire. Conclusion.

\section{A. INTRODUCTION}

Cet article résulte de la copie remaniée de notre communication présentée lors du séminaire organisé par la Konrad-Adenauer-Stiftung à Lubumbashi du 17 au 18 avril 2019. Il ne prétend pas se livrer à une étude exhaustive de la question, mais il entend cerner principalement la fonction du Maire de la Ville dans le maintien de l'ordre public dans le contexte de l'organisation politico-administrative de la RDC ainsi que les défis qu'il doit relever dans la sécurisation de son entité administrative. La communication a été présentée au moment où la Ville de Lubumbashi était exposée aux nombreux actes et généralisés d'insécurité, notamment les vols à mains armées.

La protection des personnes et de leurs biens constitue l'une des missions régaliennes de chaque Etat. Ce dernier doit pourvoir à garantir la sécurité tant à l'extérieur qu'à l'intérieur de ses frontières. La sécurité constitue avant tout un droit collectif prévu à l'article 52, al. $1^{\text {er }}$ de la Constitution du 18 février 2006 aux termes duquel « Tous les Congolais ont droit à la paix et à la sécurité tant sur le plan national qu'international ». Cette disposition est par ailleurs consacrée par l'article 23 de la Charte africaine des droits de l'homme et des Peuples ${ }^{1}$. La sécurité est assurée par la Police nationale et les Forces armées. En effet, la Police nationale est chargée de la sécurité publique, de la sécurité des personnes et de leurs

* KAZADI MPIANA Joseph, Professeur Associé à la faculté de Droit de l'Université de Lubumbashi. Courriel : Kazadimpiana@hotmail.com.

1 Art. 23, al. 1 de ladite Charte : « Les peuples ont droit à la paix et à la sécurité tant sur le plan national que sur le plan international. Le principe de solidarité et de relations amicales affirmé implicitement par la Charte de l'Organisation des Nations Unies et réaffirmé par celle de l'Organisation de l'Unité Africaine est applicable aux rapports entre les Etats... ». 
biens, du maintien et du rétablissement de l'ordre public ainsi que de la protection rapprochée des hautes autorités. ${ }^{2}$ Ces missions ont été réaffirmées par la loi organique $n^{\circ} 11 / 013$ du 11 août 2011 portant organisation et fonctionnement de la Police nationale congolaise ${ }^{3}$. La Police nationale est soumise à l'autorité civile locale et placée sous la responsabilité du ministre ayant les affaires intérieures dans ses attributions. ${ }^{4}$

Dans cette perspective le maintien de l'ordre public constitue la zone d'intersection entre le maire et la police nationale d'autant plus que l'article 42, al,2 de la Loi organique $n$ ○ 08/016 du 07 octobre 2008 portant composition, organisation et fonctionnement des Entités territoriales décentralisées et leurs rapports avec l'Etat et les provinces, énonce, parmi les attributions du maire, celle de veiller « au maintien de l'ordre public dans la ville. A cette fin, il dispose des unités de la Police nationale y affectées ». La Constitution précise, par ailleurs, en son article 184 que la Police nationale est soumise à l'autorité civile locale et elle est placée sous la responsabilité du ministère ayant les affaires intérieures dans ses attributions. La sécurité publique est parfois appelée "sûreté publique ». Celle-ci est caractérisée de manière générale par ce qui est garanti, assuré, c'est le propre d'une personne qui n'a rien à craindre ${ }^{5}$. Sa garantie suppose « la mise en œuvre de mesures capables de protéger la société contre tout ce qui menace l'intégrité de ses membres et de ses biens $»{ }^{6}$

La Constitution prescrit, en outre, que les forces armées participent, en temps de paix, à la protection des personnes et de leurs biens en apportant leur appui, s'il est requis, aux efforts déployés par la Police nationale dans le maintien de l'ordre public. Au regard de la tutelle que le Gouverneur de province exerce sur les entités territoriales décentralisées, et dans le cas d'espèce, sur les actes du Maire, ce dernier est tenu de l'informer de tout événement important survenu dans la ville et le prévenir de tout différend de nature à y troubler l'ordre public. Le ministre ayant dans ses attributions les affaires intérieures est aussi informé.

L'Etat répartit, à travers sa Constitution, ses lois et ses règlements, aux différentes autorités administratives, la charge de maintenir et d'assurer cette sécurité. Certaines de ces au-

2 Article 182 de la Constitution de la République Démocratique du Congo telle que modifiée par la Loi $n^{\circ} 11 / 002$ du 20 janvier 2011 portant révision de certains articles de la Constitution de la République Démocratique du Congo du 18 février 2006 (Textes coordonnés). Journal officiel de la République démocratique du Congo. 52è année, $\mathrm{N}^{\circ}$ spécial du 5 février 2011.

3 L'article 2 de cette loi organique dispose : « La Police nationale congolaise, ci-après la Police nationale, est un service public, civil, accessible à l'écoute de la population et chargé de la sécurité et tranquillité publiques, de la sécurité des personnes et de leurs biens, du maintien et du rétablissement de l'ordre public ainsi que de la protection rapprochée de hautes autorités. La Police nationale exerce les fonctions de la Police administrative et les fonctions de la Police judiciaire ».

4 Art. 6 de la Loi organique précitée.

5 M. DE VILLIERS et TH. DE BERRANGER (dir.), Droit public général. Paris, 2017, p. 598.

6 Idem, p. 599. 
torités administratives sont situées au niveau central ${ }^{7}$, d'autres au niveau provincial ${ }^{8}$ et puis local ${ }^{9}$. L'autorité administrative désigne à la fois un individu revêtu d'un véritable pouvoir de décision ou un organe, comprenant plusieurs personnes physiques constituées en collège, qui accomplissent des actes qui créent des droits et/ou des obligations pour les administrés ou pour d'autres agents publics ${ }^{10}$.

Dans le cadre du présent article la sécurité est envisagée dans sa dimension urbaine telle qu'elle doit être assurée par le maire de la ville qui est une autorité administrative. Son rôle ne peut être mieux analysé que si nous le plaçons dans le contexte général de l'organisation politico-administrative de la RDC ancrée dans le régionalisme couplé par la décentralisation.

\section{B. LE REGIONALISME POLITIQUE ET LA DECENTRALISATION EN RDC}

La République Démocratique du Congo s'est dotée, depuis la Constitution du 18 février 2006 de la forme d'Etat régional dans les rapports entre le pouvoir central et les provinces. Ce régionalisme politique est complété par la décentralisation dans les rapports entre le pouvoir central et les provinces d'une part avec certaines entités territoriales décentralisées (ETD) constituées par la ville, la commune, le secteur et la chefferie d'autre part. Les provinces ainsi que les ETD sont dotées de la personnalité juridique et sont gérées par les organes locaux. Elles jouissent de la libre administration et de l'autonomie de gestion de leurs ressources économiques, humaines, financières et techniques.

En examinant l'articulation du pouvoir entre le niveau central et le niveau provincial, l'on est frappé par le rapprochement de la forme de l'Etat congolais avec l'Etat fédéral. Ce rapprochement se situe dans la perspective du régionalisme politique. Par ailleurs, l'Exposé

7 Il convient de noter, de prime abord que la Constitution a déterminé que la sécurité, les affaires étrangères et la défense font partie de domaines de collaboration entre le Président de la République et le gouvernement. En outre ce dernier dispose de l'Administration publique, des Forces armées, de la Police nationale et des services de sécurité. (Art. 91 de la Constitution). Le ministère de l'Intérieur et Sécurité a, dans ses attributions la politique de la sûreté nationale, intérieure et extérieure; le maintien de l'ordre public, de la sécurité publique et la protection des personnes et de leurs biens; l'exercice du pouvoir hiérarchique sur la Police nationale et les services de sécurité; la politique de lutte contre le terrorisme (Ordonnance $n^{\circ}$ 17/025 du 10 juillet 2017 fixant les attributions des ministres. Journal officiel de la République démocratique du Congo, 19 juillet 2017, $\mathrm{N}^{\circ}$ spécial).

8 Article 63 de la loi $n^{\circ} 08 / 012$ du 31 juillet 2008 portant principes fondamentaux relatifs à la libre administration des provinces (Journal officiel de la République démocratique du Congo, Kinshasa, 31 juillet 2008) : « Le gouverneur de province représente le gouvernement central en province. Il assure, dans ce cadre, la sauvegarde de l'intérêt national, le respect des lois et règlements de la République et veille à la sécurité et à l'ordre public dans la province ».

9 A titre illustratif, le Bourgmestre assure le maintien de l'ordre public dans sa juridiction. A cette fin, il dispose des unités de la police (Article 60 de la Loi organique n ${ }^{\circ}$ 08/016 du 07 octobre 2008 portant composition, organisation et fonctionnement des Entités territoriales décentralisées et leurs rapports avec l'Etat et les provinces).

10 M. DE VILLIERS et Th. De BERRANGER, Droit public général, Paris, 2017, p.659. 
des motifs de la loi ${ }^{\circ} 08 / 012$ du 31 juillet 2008 portant Principes fondamentaux relatifs à la libre administration des provinces, est plus explicite sur cette forme d'Etat congolais : «... Le statut, l'organisation et le fonctionnement de la province procèdent des dispositions constitutionnelles qui instituent le régionalisme politique en République Démocratique du Congo, déterminent les institutions politiques de la Province et répartissent les compétences entre elle et le pouvoir central (articles 3 et 195 à 206 de la Constitution ${ }^{11}$. En outre la loi portant statut des agents de carrière des services publics de l'Etat rappelle dans son Exposé des motifs que « Depuis la promulgation de la Constitution du 18 février 2006, la République Démocratique du Congo, engagée dans le régionalisme constitutionnel, est organisée en deux niveaux du pouvoir d'Etat : d'une part, le pouvoir central exerçant la plénitude de la souveraineté étatique et, d'autre part, la province jouissant de la libre administration ainsi que de l'autonomie de gestion de ses ressources humaines, économiques, financières et techniques $»^{12}$.

Nous convenons avec Jacques DJOLI que la Constitution institue le régionalisme constitutionnel avec l'autonomie politique des provinces. Cependant, à l'intérieur des provinces, elle consacre le principe de décentralisation ${ }^{13}$. MWAYILA TSHIYEMBE voit dans le binôme régionalisme politique avec la décentralisation de l'Etat congolais la singularité de la Constitution du 18 février 2006 ${ }^{14}$. Deux lois distinctes régissent les provinces et les ETD bien qu'elles tirent leur fondement du principe de leur libre administration dans la Constitution elle-même ${ }^{15}$. Après avoir dégagé la nature de la forme de l'Etat congolais, à savoir un Etat régional dans l'articulation des rapports entre le pouvoir central et les pro-

11 Notre soulignement.

12 Loi n $^{\circ} 16 / 013$ du 15 juillet 2016 portant Statut des agents de carrière des services publics de l'Etat. Journal officiel de la république démocratique du Congo, 57è année, $\mathrm{N}^{\circ}$ spécial, Kinshasa, 3 août 2016. Notre soulignement.

13 J. DJOLI ESENG'EKELI, Droit constitutionnel. L'expérience congolaise (RDC), Paris, 2013, p. 196. Voy. aussi Voy. aussi J-M. KUMBU Ki NGIMBI, « Le statut juridique des provinces dans la Constitution congolaise du 18 février 2006 : des entités « régionalisées » dans un Etat uni(taire)? », O. NDESHYO RURIHOSE (dir.), Mélanges Célestin NGUYA-NDILA. La République démocratique du Congo : les défis récurrents de décolonisation, de l'Etat de droit et du développement économique et social, Kinshasa, 2012, pp. 943-965, spéc. à la p. 943. A. KAMUKUNY MUKINAY et J. CIHUNDA HENGELELA, « Régionalisation, décentralisation et naissance effective des vingtcinq nouvelles provinces en RD Congo : défis et perspectives de prévention des conflits », CongoAfrique, n 434, Avril 2009, pp.295 - 310, spéc. à la p. 300.

14 MWAYILA TSHIYEMBE, Quel est le meilleur système politique pour la République Démocratique du Congo : Fédéralisme, Régionalisme, décentralisation?, Paris, 2012, p. 12.

15 Loi ${ }^{\circ}$ 08/012 du 31 juillet 2008 portant Principes fondamentaux relatifs à la libre administration des provinces et la Loi organique $\mathrm{n}^{\circ} 08 / 016 \mathrm{du} 07$ octobre 2008 portant composition, organisation et fonctionnement des Entités territoriales décentralisées et leurs rapports avec l'Etat et les provinces. Ces deux lois ont été adoptées conformément aux prescrits de l'article 3 de la Constitution du 18 février 2006 qui dispose : «Les provinces et les entités territoriales décentralisées de la République Démocratique du Congo sont dotées de la personnalité juridique et sont gérées par les organes locaux. Ces entités territoriales décentralisées sont la ville, la commune, le secteur et la chefferie. Elles jouissent de la libre administration et de l'autonomie de gestion de leurs ressources 
vinces, la décentralisation prolonge cette forme régionale. La province constitue une composante politico-administrative de la République ou une entité régionalisée. Elle n'est pas prise en compte dans cet article qui se limite à développer le volet de la décentralisation.

Le principe de libre administration des collectivités territoriales, notamment les villes, les communes constitue l'une des tendances lourdes du droit constitutionnel en général et particulièrement du nouveau droit constitutionnel en Afrique. Les phénomènes de décentralisation présentent beaucoup de convergences en Afrique. La décentralisation, selon une certaine opinion, résulterait plus d'un choix imposé à l'Afrique, notamment par les bailleurs des fonds, que d'une volonté autonome des dirigeants politiques africains ${ }^{16}$. Cette affirmation, sans doute valable, ne suffit pas à rendre compte de l'option en faveur de la décentralisation et mérite d'être nuancée. Celle-ci fait l'objet des objectifs et des principes constitutionnels communs aux Etats et que même les Organisations internationales africaines, par le biais de plusieurs instruments, assignent aux Etats ${ }^{17}$.

La Conférence de l'Union africaine, lors de la tenue de sa vingt-troisième session ordinaire, avait adopté à Malabo le 27 juin 2014, la Charte africaine des valeurs et des principes de la décentralisation, de la gouvernance locale et du développement local dont les objectifs consistent, entre autres, à la promotion, à la protection et à l'encouragement de la décentralisation; la promotion et le soutien de la gouvernance locale et de la démocratie locale comme piliers de la décentralisation en Afrique (art.2 de ladite Charte). La Charte africaine de la démocratie, des élections et de la gouvernance du 30 janvier 2007 ainsi que le protocole de la CEDEAO sur la démocratie et la bonne gouvernance promeuvent les principes de décentralisation dans la gestion des Etats africains. La Charte africaine des valeurs et des

économiques, humaines, financières et techniques. La composition, l'organisation, le fonctionnement de ces entités territoriales décentralisées ainsi que leurs rapports avec l'Etat et les provinces sont fixés par une loi organique ».

16 Voy. à titre d'exemple $M$. FAU-NOUGARET, «Originalité et convergence des phénomènes de décentralisation en Afrique sub-saharienne ", disponible sur http://afrilex.u-bordeaux4.fr/sites/afrilex /IMG/pdf/doctrine-102-58.pdf consulté le 5 juin 2019; RAOGO Antoine SAWADOGO, L'État africain face à la décentralisation. La chaussure sur la tête, Paris, 2001 : A. CABANIS et M.L. MICHEL, Le constitutionnalisme de la troisième vague en Afrique francophone, Louvain-la-Neuve, 2010, notent que « cette volonté de décentralisation répond également aux injonctions des instances internationales qui, à l'aune des normes libérales, conditionnent leurs aides à l'allègement des charges publiques... », p. 161.

17 Voy. entre autres l'article 34 de la Charte africaine de la démocratie, des élections et la gouvernance du 30 janvier 2007 (en vigueur depuis le 15 février 2012) dans le cadre de l'Union africaine (Les Etats parties procèdent à la décentralisation en faveur des autorités locales démocratiquement élues conformément aux lois nationales). Notre soulignement. Dans cette même perspective nous pouvons évoquer l'article $1^{\text {er }}$, lettre d du Protocole de la CEDEAO A/SP1/12/01 sur la démocratie et la bonne gouvernance additionnel au Protocole relatif au mécanisme de prévention, de gestion, de règlement des conflits, de maintien de la paix et de la sécurité signé à Dakar le 21 décembre 2001. Parmi les principes constitutionnels communs aux membres de la CEDEAO figure, à l'article $1^{\text {er }}$, lettre d) La participation populaire aux prises de décision, le strict respect des principes démocratiques, et la décentralisation du pouvoir à tous les niveaux de gouvernement. Notre soulignement. 
principes de la décentralisation, de la gouvernance locale et du développement local adoptée à Malabo le 24 juin 2014 par la Conférence de l'Union africaine entend par décentralisation « le transfert des pouvoirs, des responsabilités, des capacités et des ressources du niveau national à tous les niveaux sous-nationaux de gouvernement afin de renforcer la capacité des gouvernements sous-nationaux à promouvoir la participation des populations et la fourniture de services de qualité $»$.

En analysant toutes les Constitutions africaines et certaines lois relatives à la décentralisation, nous pouvons affirmer que la décentralisation constitue la forme juridique de droit commun de l'Etat en Afrique. La décentralisation constitue un mode de gestion des affaires publiques largement répandue en Afrique surtout à partir des années 1990. Elle est partagée dans le monde francophone africain à telle enseigne que Jean-Nazaire TAMA conclut que la décentralisation administrative est considérée comme « un passage obligé pour tous les Etats francophones qui aspirent au développement $\rangle^{18}$. Focalisant son étude sur l'Afrique francophone de l'ouest, Ndiogou SARR souligne qu'il n'existe dans cette partie de l'Afrique un Etat dont le pouvoir n'est pas aménagé suivant le modèle de la décentralisation ${ }^{19}$.

Le fondement de la décentralisation en RDC est par conséquent constitutionnel et ne procède pas d'un transfert des compétences du pouvoir central. Comme le soutient CHABANE BENAKEZOUH, la décentralisation couplée à la libre administration perçue comme une liberté naturellement et logiquement attachée aux collectivités territoriales ne suppose pas et n'implique pas un transfert d'attribution dans la mesure où ces collectivités détiennent déjà en elles-mêmes et pour elles-mêmes des compétences qui leur sont propres ${ }^{20}$.

La Loi organique $n^{\circ}$ 08/016 du 07 octobre 2008 portant composition, organisation et fonctionnement des Entités territoriales décentralisées et leurs rapports avec l'Etat et les provinces complète les dispositions constitutionnelles en la matière. Pour renforcer davantage cette garantie des prérogatives des entités territoriales décentralisées, la Constitution interdit toute révision ayant pour objet ou effet de les réduire. La Constitution a posé le principe de la libre administration des provinces et des entités territoriales décentralisées s'inscrivant dans le nouveau droit constitutionnel des collectivités territoriales en Afrique.

Analysant le principe de libre administration des collectivités territoriales tel qu'aménagé au Maroc par la Constitution de 2011, Amal MECHERFI observe que ce principe place lesdites collectivités dans une situation d'autonomie sur le plan organique et fonctionnel. Sur le plan organique cette autonomie se manifeste par l'attribution par la Constitution à chacune des collectivités de la personnalité morale de droit public avec tous les attributs qui

18 J-N. TAMA, L'odyssée du constitutionnalisme en Afrique, Paris, 2015, p. 57.

19 N. SARR, Médiation et démocratisation. Essai sur une nouvelle technique de stabilisation due pouvoir en Afrique noire francophone, 2018, p. 300.

20 CHABANE BENAKEZOUH, «La dimension constitutionnelle des collectivités territoriales en Algérie », in GILLES J. GUGLIELMI et J. MARTIN (dir.), Le droit constitutionnel des collectivités territoriales. Aspects de droit public comparé, Boulogne-Billancourt, 2015, pp. 117-154, spéc. à la p. 138. 
s'y rattachent. Elle consiste en outre pour les collectivités territoriales de disposer d'organes élus par leurs populations, à savoir une assemblée délibérante et un exécutif local qui prennent en charge la gestion de leurs affaires. Sur le plan fonctionnel, l'autonomie se traduit d'un côté par des pouvoirs de décision propre pour tout ce qui concerne la gestion de leurs affaires leur permettant de disposer de prérogatives de puissance publique dans le respect de la constitution, de la loi et des règlements, d'autre part par des moyens suffisants (humains et matériels) dont ces collectivités peuvent disposer librement en fonction de leurs propres intérêts et dans les conditions fixées par la loi ${ }^{21}$. Pour CHABANE BENAKEZOUH, le fait d'accorder une dimension constitutionnelle aux collectivités territoriales et d'en faire des sujets de droit constitutionnel avec la décentralisation qui s'y rattache impliquent aussi bien leur valorisation et leur élévation institutionnelle afin de n'être plus phagocytées par l'Etat et ses structures déconcentrées ${ }^{22}$.

Ces considérations développées dans le cadre de l'application du principe de libre administration aux collectivités territoriales marocaines et tunisiennes, valent également, $m u$ tatis mutandis, pour les entités territoriales décentralisées de la RDC, d'autant plus que l'article 3 de la Constitution en vigueur, en son alinéa 3 est clair à ce propos ${ }^{23}$. En effet, les entités territoriales décentralisées sont la Ville, la Commune, le Secteur, et la Chefferie. Il découle de cette énumération que la Ville occupe le premier plan. D'où, l'intérêt d'examiner son statut dans l'ordonnancement juridique congolais.

\section{LE STATUT DE LA VILLE ET DU MAIRE DANS L'ORDONNANCEMENT JURIDIQUE CONGOLAIS}

La ville constitue une entité territoriale décentralisée dotée de la personnalité juridique et jouissant de la libre administration de ses ressources humaines, matérielles, financières et techniques. Le rappel du statut de la ville en RDC est fondamental pour mieux comprendre la place du maire dans le dispositif sécuritaire. Aux termes de la loi organique du 7 octobre

21 A, MECHERFI, «Les collectivités territoriales dans la Constitution marocaine : naissance du droit constitutionnel local », dans GILLES J. GUGLIELMI et J. MARTIN (dir.), Le droit constitutionnel des collectivités territoriales. Aspects de droit public comparé, Boulogne-Billancourt, 2015, pp. 59-79, spéc. à la p. 67.

22 CHABANE BENAKEZOUH, « La dimension constitutionnelle des collectivités territoriales en Algérie ", in GILLES J. GUGLIELMI et J. MARTIN (dir.), op.cit., pp. 117-154, spéc. à la p. 121.

23 Elles (provinces et Entités territoriales décentralisées) jouissent de la libre administration et de l'autonomie de gestion de leurs ressources économiques, humaines, financières et techniques. Des ouvrages ont été consacrés à la décentralisation en RDC. A titre indicatif, J-M. KUMBU KI NGIM$B I$, (dir), La décentralisation territoriale en République démocratique du Congo sous le régime de la Constitution du 18 février 2006. Bilan et perspectives, Kinshasa, 2014; B. KAIMWA MANENO, $L$ 'enjeu de la décentralisation en RDC. Organisation et gestion des entités de base : secteur et chefferie, Paris, 2014; E. KASONGO MUNGONGO, La gouvernance des entités territoriales décentralisées. Défis et enjeux de la gestion des finances publiques communales à Kinshasa, Paris, 2019; J-P. LOTOY ILANGO-BANGA, La décentralisation chaotique en République démocratique du Congo, Paris, 2018. 
2008 portant organisation, composition et fonctionnement des entités territoriales décentralisées et leurs rapports avec l'Etat et les provinces, la ville est dotée de deux organes : d'une part le conseil urbain (organe délibérant) et d'autre part le collège exécutif urbain présidé par le maire, secondé d'un adjoint et entouré de trois « ministres » urbains appelés « échevins ».

Le Maire et le Maire-adjoint sont élus au sein ou en dehors du Conseil urbain dans les conditions fixées par la loi électorale. Ils sont investis par le Ministre de la République ayant les affaires intérieures dans ses attributions dans les quinze jours de la proclamation des résultats. Passé ce délai, l'investiture est acquise de droit. ${ }^{24}$

Le Conseil urbain exerce le contrôle sur le collège exécutif urbain lequel est politiquement responsable devant ce dernier. La loi organique précitée détermine les compétences de chacun de ces deux organes. Parmi les matières d'intérêt commun urbain sur lesquelles peut délibérer le Conseil urbain figure la police des spectacles et manifestations publiques. Le Conseil urbain prend des règlements d'administration et de police. Ces règlements ne peuvent être contraires aux dispositions légales ou réglementaires édictées par l'autorité supérieure. Le Conseil sanctionne les règlements de police de peines ne dépassant pas sept jours d'emprisonnement et 25.000 Francs congolais d'amende ou d'une de ces peines seulement.

Par ailleurs, dans le cadre de cet article nous nous limitons à cerner les compétences de ces deux organes de la ville, en matière de sécurité et de maintien de l'ordre public, tout en relevant qu'en attendant l'organisation des élections urbaines qui permettront l'installation de ces deux organes, la ville est dirigée par le maire et le maire adjoint nommés par le Président de la République sur proposition du Ministre des affaires intérieures en vertu du décret-loi 082 du 2 juillet 1998 portant statut des autorités chargées de l'administration des circonscriptions territoriales. Le Ministre ayant dans ses attributions les Affaires intérieures peut, par arrêté motivé, suspendre le Maire pour compromission dans l'exercice de ses fonctions ou manquement aux devoirs de sa charge. Chaque année le gouverneur de province transmet au Ministre des Affaires intérieures un rapport d'appréciation sur les activités des bourgmestres et de leurs adjoints. En d'autres termes la loi précitée est appliquée de manière sélective dans certaines de ses dispositions en attendant son application intégrale conditionnée par l'organisation desdites élections ${ }^{25}$.

24 Article 30 de la Loi organique $n^{\circ} 08 / 016$ du 7 octobre 2008 portant composition, organisation et fonctionnement des entités territoriales décentralisées et leurs rapports avec l'Etat et les provinces (Journal officiel de la République démocratique du Congo), 49 è année, ${ }^{\circ}$ spécial, Kinshasa, 10 octobre 2008.

25 Article 126 de la loi organique précitée : «En attendant l'organisation des élections urbaines, communales et locales par la Commission électorale nationale indépendante instituée par la Constitution, les autorités des différentes entités territoriales décentralisées actuellement en poste sont gérées conformément aux dispositions du Décret-loi n 082 du 02 juillet 1998 portant statut des autorités chargées de l'administration des circonscriptions territoriales." 
Nonobstant cette application sélective de la loi organique précitée, le maintien de l'ordre public, y compris son volet sécuritaire, fait partie de prérogatives de droit commun du Maire de la ville.

\section{Le Maire face à l'impératif sécuritaire}

Examiner le rôle du maire de la ville dans le cadre de la sécurisation de son entité administrative vaut son pesant d'or, à l'aune de la décentralisation en RDC. En effet, en tant qu'autorité administrative chargée de la gestion de son entité administrative, à savoir la ville, le maire est tenu de sécuriser celle-ci et ses administrés en les rassurant de lui faire confiance. En effet la sécurisation consiste à faire passer d'un sentiment d'angoisse à un sentiment de sécurité, à une impression de confiance. La sécurité renvoie à un état d'esprit d'une personne qui se sent tranquille. Pour l'individu ou un groupe, c'est le sentiment d'être à l'abri de tout danger et risque. En déférant à cette obligation, le Maire prend des mesures de police, c'est-à-dire « des mesures coercitives susceptibles de rétablir l'ordre public, la sécurité et la tranquillité publique, et d'assurer la salubrité publique, c'est-à-dire l'hygiène publique et l'esthétique d'une agglomération donnée. Ce sont là les principaux aspects que revêt la police administrative ${ }^{26}$. Celle-ci vise « le maintien de l'ordre dans la rue, les lieux publics, la lutte contre le bruit, la prévention des accidents et fléaux, humains et naturels, les incendies, inondations, complots armés et la sauvegarde de l'hygiène publique par le contrôle de la salubrité des eaux, des denrées, la prévention des épidémies et épizooties, la lutte contre les pollutions... ». ${ }^{27}$

Or l'ordre public est traditionnellement constitué par le triptyque «sécurité, tranquillité et salubrité publiques » auquel il a été ajouté « la dignité humaine ». Ainsi l'ordre public, dont fait partie la sécurité publique, désigne, (outre des règles reconnues impératives, un Etat de paix sociale que la puissance publique cherche à maintenir (et parfois seulement à atteindre) : un bon ordonnancement ou ordre établi par la société. Cet état implique l'absence de troubles à plusieurs composantes dites matérielles et extérieures que sont la sécurité, la salubrité et la tranquillité publiques ${ }^{28}$. L'ordre public peut aussi sommairement être défini comme « une notion qui encadre et, à la fois, légitime l'action des pouvoirs publics ${ }^{29}$.

L'ordre public peut être appréhendé en deux sens. D'une part, l'ordre public concerne le maintien d'un ordre, face à certains désordres qui se traduisent par des agissements matériels. C'est l'ordre public au sens classique du maintien de la sécurité, tranquillité et salubrité publiques et des impératifs de la loi pénale. Il vient réguler les comportements par des interdictions ou des injonctions de faire ou de ne pas faire. C'est un ordre public de police

26 F.VUNDUAWE te PEMAKO, Traité de droit administratif, Bruxelles, 2007, p. 78.

27 M-C. ROUAULT, Droit administratif et institutions administratives, Bruxelles, 2018, p. 291.

28 MATHIEU TOUZEIL-DIVINA, Dictionnaire de droit public interne, Paris, 2017, p. 344.

29 G. DRAGO, "L'ordre public et la Constitution », dans Archives de philosophie du Droit, Vol. 58, 2015, pp. 199-214, spéc. à la p. 199. 
au sens le plus général et le plus classique du terme. D'autre part, une seconde conception de l'ordre public relève de techniques juridiques qui se rassemblent toutes sous une idée commune : les titulaires d'un pouvoir légal, c'est-à-dire d'un pouvoir de produire des effets de droit par leurs agissements, ne peuvent l'exercer en toute liberté car des règles s'imposent à eux dans la façon dont ils en usent ${ }^{30}$. C'est la première conception de l'ordre public qui a été prise en considération dans le cadre de cet article.

\section{CONCLUSION}

La situation généralisée de l'insécurité sur l'étendue du territoire national en général, et dans la ville de Lubumbashi en particulier, est préoccupante et interpelle les autorités administratives au premier rang desquelles figure le maire de la ville qui doit rassurer ses administrés d'être à l'abri de tout danger ou risque portant sur leur sécurité en recourant à tous les moyens de la police administrative à titre principal et subsidiairement à la police judiciaire. Bien que ces deux polices se rapprochent jusqu'à entretenir une confusion, elles se distinguent. La Police administrative constitue l'ensemble des moyens juridiques et matériels ayant pour but d'assurer le maintien de la tranquillité, de la sécurité et de la salubrité publique. $^{31}$

Par contre, lorsque l'opération de police s'inscrit dans une optique répressive après la réalisation d'une infraction, elle relève de la police judiciaire. Il convient de noter que le maire de la ville est investi, aux termes de l'article 41 de la loi organique du 7 octobre 2008 sur les ETD, de la qualité d'officier de police judiciaire à compétence générale. Le besoin de renforcer la présence des policiers dans les différents quartiers est indispensable si ces derniers peuvent inspirer la confiance auprès de la population. Il faudrait se rappeler que « l'absence de police entraîne le désordre mais trop de police tue l'ordre ". $^{32}$

Il convient de concevoir une stratégie nationale et coordonnée en matière de sécurité et dans laquelle les Maires auront leur partition à jouer en collaboration avec les conseils urbains. Cette stratégie devrait, entre autres, définir les objectifs à atteindre ainsi que les moyens matériels, logistiques, humains. Dans ce cadre la création d'une police urbaine ou municipale en nombre conséquent s'avère l'une des pistes à explorer aux fins de faire face à ce manque d'unités de Police dans les nouveaux quartiers de la ville de Lubumbashi.

Dans l'optique de rapprocher davantage l'administration des administrés le nombre actuel des communes de la ville de Lubumbashi devrait être revu à la hausse, notamment par l'éclatement de la Commune annexe dans plusieurs communes. Une nouvelle cartographie de la ville apparaît une nécessité. La population sera également partie intégrante de cette stratégie. Enfin la sécurité ne peut être garantie efficacement sans le concours d'une justice

30 G. DRAGO, "L'ordre public et la Constitution », dans Archives de philosophie du Droit, Vol. 58, 2015, pp. 199-214, spéc. à la p. 200.

31 S. GUINCHARD et TH. DEBARD, Lexique des termes juridiques, Paris, 2017.

32 M. DE VILLIERS et TH. DE BERRANGER (dir.), Droit public général. Paris, 2017, p. 591. 
à la fois rassurante et respectueuse des droits de l'homme. Il reste évident que malgré toutes les précautions, l'exigence sociale de sécurité ne semble pas tarissable. C'est qu'il y a en effet en chacun une demande de sécurité, et cette demande est adressée à la société. ${ }^{33}$

Longtemps reléguées sine die, depuis plus de dix ans, les élections urbaines demeurent incontournables afin de renforcer la légitimité du Maire et de créer les conditions de la reddition des comptes sur sa gestion auprès de ses administrés par le truchement du Conseil urbain. Cette situation rend le maire redevable à l'égard de l'autorité de tutelle (le Gouverneur de province) et des autorités hiérarchiques (Président de la République et ministre des affaires intérieures). Il ne répond pas devant ses administrés ni directement et encore moins indirectement. Il en résulte un certain déficit de légitimité du Maire en l'absence de toute organisation des élections urbaines. Et pourtant, au regard de la loi organique du 7 octobre 2008 sur les entités territoriales décentralisées, il est chef de l'exécutif urbain, appelé collège exécutif urbain politiquement responsable devant le Conseil urbain. Dans son activité de contrôle sur l'exécutif urbain, le conseil urbain peut recourir à tous les moyens, y compris la mise en cause de la responsabilité politique par le biais de la motion de censure.

Etant l'émanation du suffrage populaire, le Conseil urbain sera en mesure de mieux interpréter les besoins sécuritaires de la population et d'aider le Maire ainsi que le collège exécutif urbain à identifier les solutions les plus idoines ainsi que les moyens y afférents. Certes le défi sécuritaire ne peut être relevé de manière isolée par les Maires.

\section{BIBLIOGRAPHIE}

\section{TEXTES OFFICIELS}

Charte africaine des droits de l'homme et des Peuples

Charte africaine de la démocratie, des élections et la gouvernance du 30 janvier 2007 (en vigueur depuis le 15 février 2012)

Protocole de la CEDEAO A/SP1/12/01 sur la démocratie et la bonne gouvernance additionnel au Protocole relatif au mécanisme de prévention, de gestion, de règlement des conflits, de maintien de la paix et de la sécurité signé à Dakar le 21 décembre 2001.

Constitution de la République Démocratique du Congo telle que modifiée par la Loi n ${ }^{\circ} 11 / 002$ du 20 janvier 2011 portant révision de certains articles de la Constitution de la République Démocratique du Congo du 18 février 2006.

Loi organique $n^{\circ} 11 / 013$ du 11 août 2011 portant organisation et fonctionnement de la Police nationale congolaise

Loi organique $\mathrm{n}^{\circ} 08 / 016 \mathrm{du} 07$ octobre 2008 portant composition, organisation et fonctionnement des Entités territoriales décentralisées et leurs rapports avec l'Etat et les provinces).

33 Idem, p. 598. 
Loi organique $\mathrm{n}^{\circ}$ 08/016 du 7 octobre 2008 portant composition, organisation et fonctionnement des entités territoriales décentralisées et leurs rapports avec l'Etat et les provinces (Journal officiel de la République démocratique du Congo), 49 è année, nº spécial, Kinshasa, 10 octobre 2008.

Loi ${ }^{\circ}$ 16/013 du 15 juillet 2016 portant Statut des agents de carrière des services publics de 1'Etat. Journal officiel de la république démocratique du Congo, 57è année, $\mathrm{N}^{\circ}$ spécial, Kinshasa, 3 août 2016.

Loi n ${ }^{\circ}$ 08/012 du 31 juillet 2008 portant principes fondamentaux relatifs à la libre administration des provinces (Journal officiel de la République démocratique du Congo, Kinshasa, 31 juillet 2008)

Ordonnance $\mathrm{n}^{\circ}$ 17/025 du 10 juillet 2017 fixant les attributions des ministres. Journal officiel de la République démocratique du Congo, 19 juillet 2017, $\mathrm{N}^{\circ}$ spécial.

Décret-loi n082 du 02 juillet 1998 portant statut des autorités chargées de l'administration des circonscriptions territoriales."

\section{LES OUVRAGES}

A. CABANIS et M.L. MICHEL, Le constitutionnalisme de la troisième vague en Afrique francophone, Louvain-la-Neuve, 2010

B. KAIMWA MANENO, L'enjeu de la décentralisation en RDC. Organisation et gestion des entités de base : secteur et chefferie, Paris, 2014

F.VUNDUAWE te PEMAKO, Traité de droit administratif, Bruxelles, 2007

J. DJOLI ESENG'EKELI, Droit constitutionnel. L'expérience congolaise (RDC), Paris, 2013

$J-M$. KUMBU KI NGIMBI, (dir), La décentralisation territoriale en République démocratique du Congo sous le régime de la Constitution du 18 février 2006. Bilan et perspectives, Kinshasa, 2014

J-N. TAMA, L'odyssée du constitutionnalisme en Afrique, Paris, 2015

J-P. LOTOY ILANGO-BANGA, La décentralisation chaotique en République démocratique du Congo, Paris, 2018.

KASONGO MUNGONGO, La gouvernance des entités territoriales décentralisées. Défis et enjeux de la gestion des finances publiques communales à Kinshasa, Paris, 2019

M. DE VILLIERS et TH. DE BERRANGER (dir.), Droit public général. Paris, 2017

M. DE VILLIERS et TH. DE BERRANGER (dir.), Droit public général. Paris, 2017

MATHIEU TOUZEIL-DIVINA, Dictionnaire de droit public interne, Paris, 2017

M-C. ROUAULT, Droit administratif et institutions administratives, Bruxelles, 2018

MWAYILA TSHIYEMBE, Quel est le meilleur système politique pour la République Démocratique du Congo : Fédéralisme, Régionalisme, décentralisation?, Paris, 2012

N. SARR, Médiation et démocratisation. Essai sur une nouvelle technique de stabilisation due pouvoir en Afrique noire francophone, 2018

RAOGO Antoine SAWADOGO, L'État africain face à la décentralisation. La chaussure sur la tête, Paris, 2001

S. GUINCHARD et TH. DEBARD, Lexique des termes juridiques, Paris, 2017 


\section{LES ARTICLES ET REVUES}

A, MECHERFI, «Les collectivités territoriales dans la Constitution marocaine : naissance du droit constitutionnel local », dans GILLES J. GUGLIELMI et J. MARTIN (dir.), Le droit constitutionnel des collectivités territoriales. Aspects de droit public comparé, Boulogne-Billancourt, 2015, pp. 59-79, spéc. à la p. 67.

A. KAMUKUNY MUKINAY et J. CIHUNDA HENGELELA, « Régionalisation, décentralisation et naissance effective des vingt-cinq nouvelles provinces en RD Congo: défis et perspectives de prévention des conflits », Congo-Afrique, n ${ }^{\circ} 34$, Avril 2009, pp.295 - 310.

CHABANE BENAKEZOUH, « La dimension constitutionnelle des collectivités territoriales en Algérie », in GILLES J. GUGLIELMI et J. MARTIN (dir.), Le droit constitutionnel des collectivités territoriales. Aspects de droit public comparé, Boulogne-Billancourt, 2015, pp. 117-154.

CHABANE BENAKEZOUH, « La dimension constitutionnelle des collectivités territoriales en Algérie », in GILLES J. GUGLIELMI et J. MARTIN (dir.), op.cit., pp. 117-154.

DRAGO, "L'ordre public et la Constitution », dans Archives de philosophie du Droit, Vol. 58, 2015, pp. 199-214.

DRAGO, "L'ordre public et la Constitution », dans Archives de philosophie du Droit, Vol. 58, 2015, pp. 199-214.

J-M. KUMBU Ki NGIMBI, « Le statut juridique des provinces dans la Constitution congolaise du 18 février 2006 : des entités « régionalisées » dans un Etat uni(taire)? », O. NDESHYO RURIHOSE (dir.), Mélanges Célestin NGUYA-NDILA. La République démocratique du Congo : les défis récurrents de décolonisation, de l'Etat de droit et du développement économique et social, Kinshasa, 2012, pp. 943-965.

M. FAU-NOUGARET, «Originalité et convergence des phénomènes de décentralisation en Afrique sub-saharienne », disponible sur http://afrilex.u-bordeaux4.fr/sites/afrilex/IMG/pdf/doctrine-102-5 8.pdf consulté le 5 juin 2019; 